\title{
Pericardial effusion unrelated to surgery is a predictor of mortality in heart transplant patients
}

\author{
Simon F. Stämpfli ${ }^{1 *}$, Tardu Özkartal ${ }^{1 *}$, Niels Hagenbuch ${ }^{2}$, Stephan Bernhart ${ }^{1}$, \\ Andreas J. Flammer ${ }^{1}$, Alessandra Vecchiati ${ }^{1}$, Georg M. Fröhlich ${ }^{1}$, \\ Frank Ruschitzka ${ }^{1}$, Leonhard Held ${ }^{2}$, Felix C. Tanner ${ }^{1}$ \\ ${ }^{1}$ Department of Cardiology, University Heart Center Zurich, Switzerland \\ ${ }^{2}$ Department of Biostatistics; Epidemiology, Biostatistics and Prevention Institute, \\ University of Zurich, Switzerland
}

\begin{abstract}
Background: Hemodynamically irrelevant pericardial effusion (PeEf) is a predictor of adverse outcome in heart failure patients. The clinical relevance of a PeEf unrelated to surgery in heart transplant patients remains unknown. This study assesses the prognostic value of PeEfoccurring later than 1 year after transplantation. Methods: All patients undergoing heart transplantation in Zurich between 1989 and 2012 were screened. Cox proportional hazard models were used to analyze mortality (primary) and hospitalization (secondary endpoint). PeEf time points were compared to baseline for rejection, immunosuppressants, tumors, inflammation, heart failure, kidney function, hemodynamic, and echocardiographic parameters.

Results: Of 152 patients (mean age $48.3 \pm 11.9$ ), 25 developed PeEf. Median follow-up period was 11.9 (IQR 5.8-17) years. The number of deaths was 6 in the PeEf group and 46 in the non-PeEf group. The occurrence of PeEf was associated with a 2.5-fold increased risk of death (HR 2.49, 95\% CI 1.02-6.13, $p=0.046$ ) and hospitalization (HR 2.53, 95\% CI 1.57-4.1, $p=0.0002$ ).

Conclusions: This study reveals that the finding of hemodynamically irrelevant PeEf in heart transplant patients is a predictor of adverse outcome, suggesting that a careful clinical assessment is warranted in heart transplant patients exhibiting small PeEf. (Cardiol J 2018; 25, 6: 714-721)

Key words: survival, hospitalization, cancer, prognosis, echocardiography
\end{abstract}

\section{Introduction}

Pericardial effusion (PeEf) is a common finding in the acute phase after heart transplantation [1]. During the first months, moderate to severe PeEf has been described in up to $21 \%$ of patients and has been subject of several studies [2-4]. In contrast, the development of PeEf later than 1 year after heart transplantation is rare [5] and its clinical significance has not been investigated.

Small and hemodynamically irrelevant PeEf, which may be observed during routine echocardiography [5], is associated with increased mortality in patients with chronic heart failure [6] as well as in those with pulmonary hypertension [7]. How- ever, in patients after heart transplantation, the prognostic value of a hemodynamically irrelevant PeEf not related to surgery remains undetermined. Thus, the aim of this study was to assess the prognostic significance of this finding in patients 1 year or later after heart transplantation.

\section{Methods}

Patient population and baseline characteristics

All patients undergoing heart transplantation at the University Heart Center Zurich between August 1989 and July 2012 were retrospectively screened for inclusion in this study. Exclusion

Address for correspondence: Felix C. Tanner, MD, Department of Cardiology, University Heart Center Zurich,

Rämistrasse 100, CH-8091 Zurich, Switzerland, tel: +41 44255 15 15, fax: +41 4425587 01, e-mail: felix.tanner@usz.ch

Received: 04.10.2017 Accepted: 29.12.2017

*These authors contribute equally to this work. 
criteria were death within the first year after transplantation, age $<16$ years at heart transplantation, and lack of echocardiography studies. Baseline was defined as the time of the first echocardiography study later than 1 year after heart transplantation. Data on age, gender, body weight, blood pressure, heart rate, primary heart disease (ischemic vs. non-ischemic), New York Heart Association (NYHA) functional class, International Society for Heart and Lung Transplantation (ISHLT) score, medication, and laboratory values were retrieved from local databases and patient records. Survival and hospitalization data was retrieved from the post-transplant database and completed by detailed reviewing of electronic medical records as well as paper charts from the heart transplantation unit of the University Heart Center Zurich. The study was approved by the local ethical committee and complies with the Declaration of Helsinki. Informed consent was obtained from all subjects.

\section{Study groups and outcome measures}

Patients with no signs of PeEf were defined as the control group. Patients exhibiting a PeEf were assigned to the PeEf group at the first time point the PeEf was detected and remained in this group until the end of the study. Hence, with each occurrence of a PeEf, the composition of the two groups changes, as a patient moves from the control to the PeEf group. The primary outcome measure was allcause mortality. The secondary outcome measure was unscheduled hospitalization for any cause.

\section{Echocardiography}

All echocardiograms available were included in the analysis and were analyzed independently by two experienced physicians. In general, echocardiograms were performed when applicable every 2 years, additionally whenever clinically indicated. The presence of a hemodynamically irrelevant PeEf was defined as an end-diastolic hypoechogenic space in the pericardium without signs of hemodynamic compromise (inversion or collapsing of right side heart chambers, abnormal ventricular septal motion, respiratory variation of mitral inflow $>25 \%$ ). Other echocardiographic parameters were analyzed according to guidelines of the European Society of Cardiology [8] including grading of mitral and tricuspid regurgitation, tricuspid annular plane systolic excursion (TAPSE), mitral annular plane systolic excursion, left ventricular end-diastolic volume and ejection fraction, end-diastolic diameter, end-systolic diameter, left ventricular mass index, left atrial end-systolic diameter, right ventricular (RV) end-diastolic diameter, right atrial dimensions (RA long and short axis), fractional area change, and systolic RV-RA pressure gradient ( $\Delta \mathrm{p} R \mathrm{R}-\mathrm{RA})$.

\section{Laboratory and histological parameters}

Blood samples were taken during clinical routine follow-up (usually every 6 months in the heart failure and heart transplantation clinic) as well as during unscheduled hospitalization. C-reactive protein and creatinine levels were analyzed using the Cobas analyser (Roche Diagnostics, Basel, Switzerland) at the Institute of Clinical Chemistry of the University Hospital Zurich.

Right ventricular septal endomyocardial biopsies were performed according to the post-transplant surveillance program of the documented heart transplantation clinic and cardiac cellular allograft rejection grading was performed according to the ISHLT guidelines [9]. In general, endomyocardial biopsies were performed when applicable every 6 months, additionally when otherwise indicated.

\section{Statistical analysis}

Statistical analysis was performed by the Epidemiology, Biostatistics and Prevention Institute in the Department of Biostatistics at the University of Zurich. The time-dependent variable PeEf was taken into account as described in Anderson et al. 1983 [10] ("Mantel-Byar Approach") and Andersen 1992 [11], and modelled with a Cox regression with age as time scale and with or without adjustment for age at transplantation and gender. Transition from non-PeEf to PeEf group was taken into account by creating a data set listing the time-dependent group variable for each follow-up visit of patients and the time span during which the assignment to a group did not change. In this model, all patients belong to the group without $\mathrm{PeEf}$ at the beginning of the observation and shift to the other group, as soon as they develop PeEf. Thus, groups are not constant and direct statistical comparison of the groups with the final distribution was not performed. Statistical analysis of not normally distributed parameters at baseline vs. time of PeEf was performed using the Wilcoxon signedrank test. A p-value $<0.05$ was considered statistically significant. As statistical software, the R programming language [12] Version 3.4.1 was applied.

\section{Results}

\section{Patient population}

and echocardiography findings

All 313 patients undergoing heart transplantation at the University Heart Center Zurich between 
August 1989 and July 2012 were evaluated for this study. Exclusion criteria were death within the first year after transplantation (64 patients), age $<16$ years at heart transplantation (8 patients), and lack of echocardiography studies (89 patients, mostly due to follow up in other hospitals). A total of 152 patients were included in the study (Suppl. Fig. 1). Clinical baseline characteristics of the study population are displayed in Table 1. Most of the patients were male $(84.9 \%)$ and had transplants due to non-ischemic heart disease $(65 \%)$. Mean age at transplantation was $48.3 \pm 11.9$ years. Median follow-up duration was 11.9 (interquartile range [IQR] 5.8-17) years.

Baseline echocardiography was performed after a median of 3 (IQR 1.5-9.5) years post heart transplantation. Echocardiographic baseline characteristics are displayed in Table 1 . Moderate or severe tricuspid regurgitation was frequent $(\mathrm{n}=22$, $15.2 \%)$. Twenty-five patients developed PeEf over time with a PeEf incidence of 14.4 per 1000 patient-years. First diagnosis of PeEf occurred after a median of 11.1 (IQR 4.1-14) years after heart transplantation. Hemodynamically relevant PeEf did not occur in any patients. Except for one, all PeEf were small with a maximal width of $1 \mathrm{~cm}$ in diastole. The one large (but also hemodynamically irrelevant) PeEf exhibited spontaneous regression without recurrence. At the time of occurrence, broad examinations revealed that it was most likely triggered by a viral polyserositis.

Direct comparison of patient groups was not legitimate since patients exhibiting a PeEf were assigned to the PeEf group at the first time point when PeEf was detected and remained in this group until the end of the study. Hence, with each occurrence of PeEf, the composition of the two groups changed (as described in the methods section).

\section{Mortality}

The total number of deaths was 6 in patients with PeEf and 46 in those without PeEf. When adjusted for age at transplantation and gender, the occurrence of PeEf was associated with a 2.5 -fold increase in the risk of death (adjusted hazard ratio [HR] 2.49, 95\% confodence interval [CI] 1.02-6.13, $\mathrm{p}=0.046$ ). In the unadjusted analysis, there was a trend towards increased mortality (unadjusted HR 2.27, 95\% CI 0.95-5.47, $\mathrm{p}=0.066$ ). The probability of survival for patients with PeEf as compared to patients never developing PeEf is depicted in Figure 1A (for demonstration purposes, the time-point of first PeEf is hypothetically set to baseline).
Table 1. Baseline characteristics of all patients.

\begin{tabular}{|c|c|c|}
\hline & Baseline & $\mathbf{n}$ \\
\hline \multicolumn{3}{|l|}{ Clinical parameters } \\
\hline Age [years] & $48.3 \pm 11.9$ & 152 \\
\hline Male & $129(84.9 \%)$ & 152 \\
\hline BMI $\left[\mathrm{kg} / \mathrm{m}^{2}\right]$ & $26.3 \pm 5.1$ & 145 \\
\hline Systolic BP [mmHg] & $136.5 \pm 15.4$ & 28 \\
\hline Heart rate [bpm] & $85.7 \pm 13.1$ & 139 \\
\hline IHD & $53(34.9 \%)$ & 152 \\
\hline Non-IHD & $99(65.1 \%)$ & 152 \\
\hline \multicolumn{3}{|l|}{ Echocardiography } \\
\hline LVEF [\%] & $60.9 \pm 7.4$ & 122 \\
\hline LV EDV [mL] & $89.7 \pm 23.4$ & 98 \\
\hline LV EDD [mm] & $47 \pm 5.1$ & 135 \\
\hline LV ESD [mm] & $29 \pm 5.5$ & 134 \\
\hline LVMI $\left[\mathrm{g} / \mathrm{m}^{2}\right]$ & $98.4 \pm 27.7$ & 102 \\
\hline LA ESD [mm] & $48 \pm 10$ & 136 \\
\hline MAPSE [mm] & $15.6 \pm 2.8$ & 60 \\
\hline $\begin{array}{l}\text { Mitral regurgitation: } \\
\geq \text { moderate }\end{array}$ & $1(0.7 \%)$ & 143 \\
\hline TR: moderate & $13(9.0 \%)$ & 145 \\
\hline TR: severe & $9(6.2 \%)$ & 145 \\
\hline FAC [\%] & $43.2 \pm 9.3$ & 51 \\
\hline TAPSE [mm] & $18.4 \pm 4.3$ & 66 \\
\hline RVEDD [mm] & $32 \pm 6.5$ & 94 \\
\hline RA long axis [mm] & $53 \pm 9$ & 124 \\
\hline RA short axis [mm] & $39 \pm 7.5$ & 119 \\
\hline$\Delta \mathrm{p}$ RV-RA [mmHg] & $27.1 \pm 8.1$ & 117 \\
\hline
\end{tabular}

Values presented as mean mean \pm standard deviation or number and percentage; $\mathrm{BMI}$ - body mass index; $\mathrm{BP}$ - blood pressure; EDD - end-diastolic diameter; EDV - end-diastolic volume; ESD - end-systolic diameter; FAC - fractional area change; IHD - ischemic heart disease; LA - left atrium; LV - left ventricle; LVEF - left ventricular ejection fraction; LVMI - left ventricular mass index; MAPSE - mitral annular plane systolic excursion; RA — right atrium; RV — right ventricle; TAPSE — tricuspid annular plane systolic excursion; TR - tricuspid regurgitation; $\Delta \mathrm{p}$ - pressure gradient

Causes of death are depicted in Figure 1B. Cancer was the main reason for death $(\mathrm{n}=15,28.8 \%)$, followed by infection $(\mathrm{n}=8,15.4 \%)$, heart failure ( $\mathrm{n}=7,13.4 \%)$, and cardiac allograft vasculopathy $(\mathrm{n}=4,7.7 \%)$. In $2(3.8 \%)$ patients, rejection, bleeding, or renal failure was the cause of death, respectively. Other causes of death included 2 perioperative complications, 1 documented arrhythmia, 1 pulseless electrical activity, and 1 stroke. In 7 patients, death occurred for unknown reasons.

\section{Hospitalization}

The absolute number of unscheduled hospitalizations was 42 in patients with PeEf and 136 in 


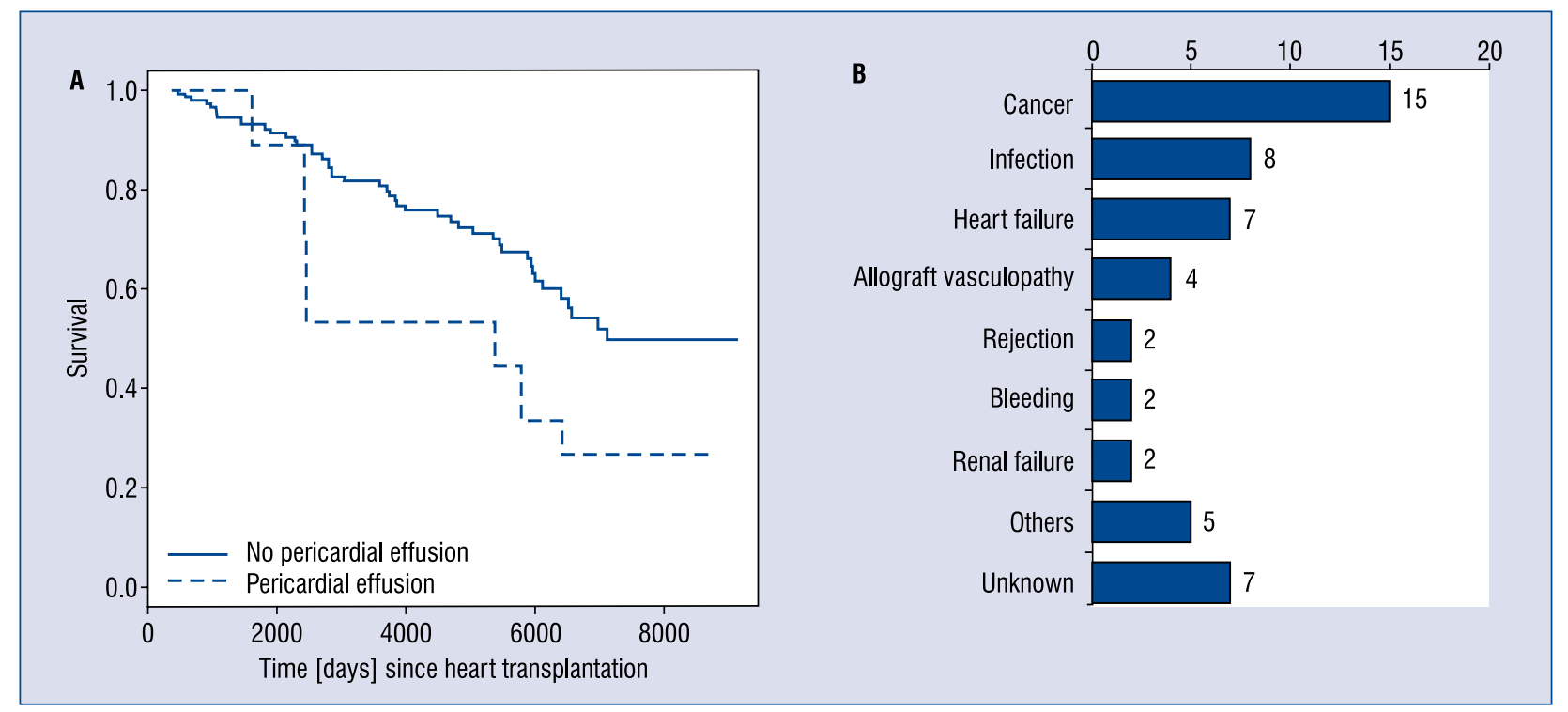

Figure 1. A. Separate estimated survivor functions for patients after heart transplantation. The solid black line is the survivor function for patients without pericardial effusion; the broken line is for patients who showed at least once a pericardial effusion (for demonstration purposes, the time-point of first pericardial effusion is hypothetically set to baseline); B. Cause of death in all patients (52 deaths).

patients without PeEf. Median interval between transplantation and the first unscheduled hospitalization was 5.6 (IQR 3-10) years. Median duration of hospitalization was 8 (IQR 6-15) days. In a Cox proportional hazard model, the risk of being admitted for hospitalization was significantly increased in patients exhibiting a prior PeEf compared to patients without PeEf (unadjusted HR 2.53, 95\% CI 1.57-4.1, $\mathrm{p}=0.0002$ ). Figure $2 \mathrm{~A}$ compares the probability of freedom from unscheduled hospitalization in patients with PeEf with patients never developing PeEf (for demonstration purposes, the time-point of first PeEf is hypothetically set to baseline).

The main reason for hospitalization (Fig. 2B) was infection ( $\mathrm{n}=137,47.6 \%)$, followed by a heterogeneous group of predominantly arterial and venous cardiovascular problems $(\mathrm{n}=30,10.4 \%)$ and cancer $(n=20,6.9 \%)$. No hospitalizations were because of pericardial effusion.

\section{Characterization of patients at the time of pericardial effusion}

To evaluate possible causes of PeEf, patients developing PeEf were characterized in more detail. Paired analysis for comparison of various parameters at the time of PeEf versus baseline revealed no difference for most parameters (Table 2). In particular, rejection stage, inflammation parameters, renal function, left ventricular function, right ventricular dimensions and tricuspid regurgitation were comparable at both time points.

NYHA functional class exhibited a trend towards higher stages at the time of PeEf as compared to baseline (median 1 IQR $1-1.875$ vs. 2 IQR $1.125-2, \mathrm{p}=0.053, \mathrm{n}=14$ ), whereas the N-terminal fragment of prohormone B-type natriuretic peptide (NT-proBNP) did not differ significantly. Left atrial size as assessed by left atrial-end-systolic diameter was larger at the time of PeEf (median 4.05 IQR $3.75-5.1$ vs. 5.2 IQR $4.38-5.55 \mathrm{~cm}$, $\mathrm{p}=0.021, \mathrm{n}=12$ ). Systolic blood pressure was reduced at the time of PeEf (median 136.5 IQR $130-142$ vs. 117.5 IQR $111.2-128 \mathrm{mmHg}$; $=0.004$, $\mathrm{n}=14$ ). Longitudinal shortening of the right ventricle as assessed by TAPSE was also decreased under these conditions (median 18 IQR 18-22 vs. 17 IQR $15-19 \mathrm{~mm}, \mathrm{p}=0.033, \mathrm{n}=7$ ).

The temporal relationship between the detection of PeEf and the time of transplantation was also evaluated. There was a linear increase in the number of patients affected by a PeEf with time after transplantation. No association pattern was detectable regarding the incidence of $\mathrm{PeEf}$ and the time after transplantation (Fig. 3A). Similarly, the incidence of malignant tumors was examined in relation to the time-point of PeEf. No association was evident between the incidence of malignant tumors and that of PeEf (Fig. 3B). Immunosuppres- 


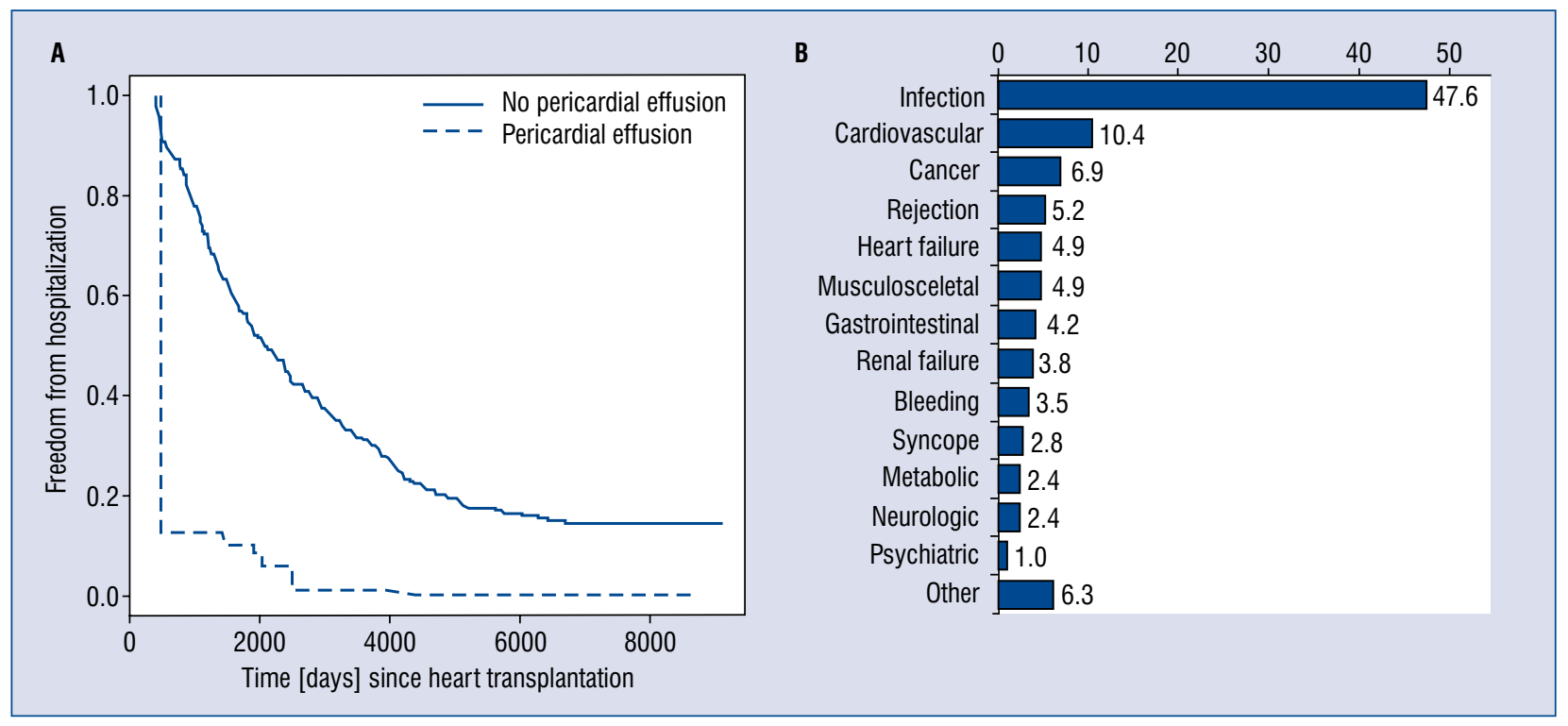

Figure 2. A. Separate estimated functions depicting freedom from unscheduled admission to a hospital for patients after heart transplantation. The solid black line is the function for patients without pericardial effusion; the broken line is for patients who showed at least once a pericardial effusion (for demonstration purposes, the time-point of first pericardial effusion is hypothetically set to baseline); B. Cause of hospitalization in all patients (137 hospitalizations).

Table 2. Analysis of parameters at baseline vs. time of pericardial effusion (PeEf) in patients developing a PeEf and having the parameters assessed at both time points.

\begin{tabular}{|c|c|c|c|c|}
\hline & Baseline & Time of PeEf & $\mathbf{P}$ & $\mathbf{n}$ \\
\hline \multicolumn{5}{|l|}{ Rejection } \\
\hline ISHLT stage & $1.0(0.0-1.0)$ & $1.0(0.0-1.0)$ & NS & 13 \\
\hline \multicolumn{5}{|c|}{ Inflammation and kidney function } \\
\hline CRP [mg/L] & $3.0(1.50-10.55)$ & $19.0(3.05-67.00)$ & NS & 15 \\
\hline Leucocytes [G/L] & $6.75(4.94-8.16)$ & $6.34(5.02-8.15)$ & NS & 17 \\
\hline Creatinine $[\mu \mathrm{mol} /]$ & $156(142-179)$ & $131(104-219)$ & NS & 17 \\
\hline \multicolumn{5}{|l|}{ Heart failure } \\
\hline NYHA class [1-4] & $1.00(1.00-1.875)$ & $2.00(1.125-2.00)$ & 0.053 & 14 \\
\hline Body weight [kg] & $79.0(68.3-89.8)$ & $76.0(67.0-89.0)$ & NS & 16 \\
\hline NT-proBNP [ng/L] & 465 (146-1577) & $534(166-2555)$ & NS & 13 \\
\hline \multicolumn{5}{|l|}{ Blood pressure and heart rate } \\
\hline Systolic BP [mmHg] & $136.5(130.0-142.0)$ & $117.5(111.2-128.0)$ & 0.004 & 14 \\
\hline Heart rate $[\mathrm{bpm}]$ & $91(80-98)$ & $88(79-91)$ & NS & 14 \\
\hline \multicolumn{5}{|l|}{ Echocardiography } \\
\hline LVEF [\%] & $62.5(60.0-64.0)$ & $61.5(59.0-64.8)$ & NS & 14 \\
\hline LAESD [cm] & $4.05(3.75-5.10)$ & $5.20(4.38-5.55)$ & 0.021 & 12 \\
\hline Mitral regurgitation (1-4) & $1.0(1.0-1.0)$ & $1.0(1.0-2.0)$ & NS & 14 \\
\hline Tricuspid regurgitation (1-4) & $1.0(1.0-2.0)$ & $2.0(1.0-2.0)$ & NS & 16 \\
\hline$\Delta \mathrm{p}$ RV-RA [mmHg] & $25.0(20.0-28.5)$ & $28.0(22.0-29.0)$ & NS & 11 \\
\hline TAPSE [mm] & $18(18-22)$ & $17(15-19)$ & 0.033 & 7 \\
\hline
\end{tabular}

Values are presented as median $\left(1^{\text {st }}-3^{\text {rd }}\right.$ quartile). BP — blood pressure; CRP — C-reactive protein; ISHLT — International Society for Heart and Lung Transplantation; LAESD — left atrial end-systolic diameter; LVEF — left ventricular ejection fraction; NT-proBNP — N-terminal fragment of prohormone B-type natriuretic peptide; NYHA — New York Heart Association; RA — right atrium; RV — right ventricle; TAPSE — tricuspid annular plane systolic excursion; $\Delta \mathrm{p}$ - pressure gradient 


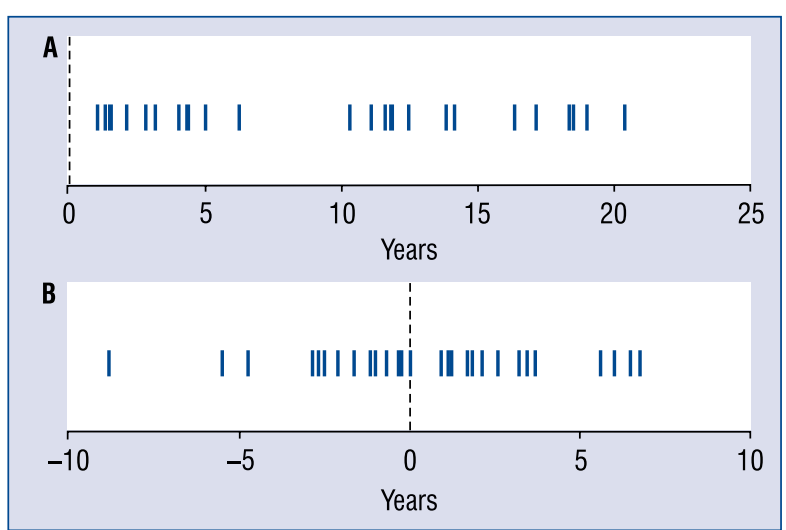

Figure 3. A. Diagnosis of pericardial effusion (PeEf) (blue lines) and its temporal relation to heart transplantation (dotted line) in all patients with PeEf; 25 patients, 30 PeEf; B. Diagnosis of malignant tumors (blue lines) and its temporal relation to first PeEf (dotted line) in all patients with PeEf and tumor diagnosis; 15 patients, 30 tumors.

Table 3. Specific immunosuppressant therapy at baseline versus at time of pericardial effusion (PeEf) (all patients who developed a PeEf).

\begin{tabular}{lcc}
\hline Medication & Baseline & Time of PeEf \\
\hline Ciclosporin & $12(71 \%)$ & $15(60 \%)$ \\
Azathioprine & $11(65 \%)$ & $13(52 \%)$ \\
Cortisone & $9(53 \%)$ & $12(48 \%)$ \\
Mycophenolic acid & $6(35 \%)$ & $10(40 \%)$ \\
Tacrolimus & $5(29 \%)$ & $6(24 \%)$ \\
Everolimus & $2(12 \%)$ & $1(4 \%)$ \\
Sirolimus & $0(0 \%)$ & $3(12 \%)$ \\
\hline
\end{tabular}

sant medication was comparable at baseline and time of PeEf (Table 3). Sirolimus was prescribed in 3 patients at the time of PeEf whereas it was not prescribed at baseline.

\section{Discussion}

This study reveals that the presence of a hemodynamically irrelevant PeEf occurring unrelated to surgery in heart transplant patients is a predictor of mortality and unscheduled hospitalization. PeEf occurring during the first year after heart transplantation were not included in this analysis since PeEf in this period is usually related to surgery, resolves spontaneously $[2,13]$ usually within 3 months, and is associated with recipient-donor weight mismatch and possibly rejection $[1-3,14,15]$.

In this study, the incidence of PeEf was 14.4 per 1000 patient-years during follow-up. No association of PeEf with the time after transplantation was observed. This is in contrast to another study demonstrating a continuous decrease in the incidence of PeEf after transplantation [5]. In that study, however, the first year after transplantation was included and the average follow-up was no more than 3 years, suggesting that many surgery-related PeEf were considered. This type of PeEf is a common finding and associated with transplantation-independent surgical risk [16]. In the present population, however, about half of the PeEf not related to surgery occurred more than 10 years after transplantation.

It was observed that patients with PeEf were at a 2.5 -fold higher risk of death than those remaining free of PeEf. This is in line with studies assessing the prognostic value of PeEf in patients with heart failure [6] or pulmonary hypertension [7]. In patients with or without PeEf, the main causes of death were cancer and infections, followed by heart failure and cardiac allograft vasculopathy. This is consistent with the latest report of The Registry of the International Society for Heart and Lung Transplantation reporting malignant tumors, rejection-independent graft failure, infection, cardiac allograft vasculopathy, and failure of other organs as the main reasons for longterm mortality in heart transplant recipients [17]. These findings suggest that the PeEf observed in the present population might not be associated with a specific pathologic condition but rather be a general prognostic indicator for a worse clinical course.

To further characterize the prognostic value of small PeEf, hospitalization rates were studied. The hazard of being hospitalized was 2.5-fold higher in patients diagnozed with PeEf at any time compared to patients without PeEf. The steep decrease (Fig. 2A) in the probability for remaining free of hospitalization in the PeEf group early during observation is mainly driven by a single patient who had a PeEf documented on day 485 and was subsequently hospitalized three times in rapid succession due to causes not related to PeEf. Exclusion of this patient, however, did not alter the results significantly (HR 2.24, 95\% CI 1.33-3.77). Infection was the reason for almost half of hospitalizations, followed by cardiovascular complications, cancer, and rejection. Similar to these findings, infections have been reported to be the leading cause for hospitalization during the first 5 years after transplantation [17]. 
To evaluate possible causes for the development of PeEf, patients with PeEf were analyzed in detail. At the time-point of PeEf no difference was observed when compared to baseline for most parameters such as rejection stage, inflammation parameters, renal function, left ventricular function, right ventricular size, and tricuspid regurgitation. NYHA functional class and left atrial size, however, were elevated, whereas NT-proBNP remained unchanged. This discrepancy may be due to the fact that the NYHA classification evaluates the occurrence of symptoms which are often, but not exclusively, due to heart failure [18]. Several studies showed that different comorbidities like depression [19], anaemia, chronic obstructive pulmonary disease, and others [20] can significantly reduce NYHA functional class. Thus, alterations in NYHA functional class can be multifactorial and are partially driven by a patient's general condition. The increase in left atrial size may represent elevated filling pressures, even though it is not solely dependent on diastolic pressure, particularly in transplanted hearts. Interestingly, systolic blood pressure was reduced at the time of PeEf. It is well known, that a reduced systolic blood pressure is associated with negative prognosis in multiple clinical settings, such as heart failure [21], infection [22], pulmonary embolism [23], and myocardial infarction [24]. In light of this, the differences in NYHA class and blood pressure are most likely multifactorial and may be considered a marker for increased morbidity in general. The reduction in TAPSE was also significant. However, in contrast to the other parameters quantitative change and patients having measured TAPSE at both time points was low, so that it seems clinically negligible. As expected in transplanted patients, relevant tricuspid regurgitation was more frequent in patients having a PeEf at a later stage ( $>12$ years after transplantation), suggesting that in these situations tricuspid dysfunction may be more relevant in contributing to PeEf than in patients at earlier stages.

Hypothesizing that PeEf could be related to malignancy, analysis of the temporal context of $\mathrm{PeEf}$ and the time of diagnosis of malignant tumors was also performed. However, no association pattern was evident, suggesting that even though cancer was the leading cause of death in this population, most PeEf were not of malignant origin. Similarly, C-reactive protein was comparable at both time points (baseline and PeEf), even though infection was the second most common cause of death. Hence, neither tumors nor infections seem to induce a PeEf in a direct manner, again pointing to the interpretation that the occurrence of a small $\mathrm{PeEf}$ in a transplant recipient is a general marker of increased mortality and morbidity.

Immunosuppressant medication was comparable during baseline and at the time of PeEf with the exception of sirolimus. The latter was described to be associated with PeEf in a small study (4 PeEf in 25 patients) [25]. In the present population, it was part of the medication in 3 patients with PeEf whereas it was not prescribed at baseline. Hence, sirolimus may at least in part account for the PeEf in these 3 patients.

Taking all these data into account, this study suggests that the etiology of PeEf is variable and may often be multifactorial (heart failure, malignancy, infection, and others), suggesting that the finding of PeEf in a heart transplant patient should lead to further investigation for excluding potentially treatable pathologies not necessarily related to cardiac function per se.

\section{Limitations of the study}

Given the observational, retrospective study design, the limitation of this study is a possible confounding bias (taking into account that echocardiograms were performed during regular follow-up as well as for clinical reasons) additionaly the problem of informative but missing measurements of patients not requiring medical care or those being examined in other hospitals.

\section{Conclusions}

In summary, this study reveals that the echocardiographic finding of a hemodynamically irrelevant PeEf in heart transplant patients is a predictor of adverse outcome, evidenced by a 2.5 times higher risk of both death and hospitalization. Thus, a small PeEf — which may be observed during routine echocardiography — should be regarded as a "red flag", leading to further examination of the patient with a focus on potentially treatable causes not directly related to the pericardial effusion.

\section{Conflict of interest: None declared}

\section{References}

1. Vandenberg BF, Mohanty PK, Craddock KJ, et al. Clinical significance of pericardial effusion after heart transplantation. J Heart Transplant. 1988; 7(2): 128-134, indexed in Pubmed: 3284984.

2. Quin JA, Tauriainen MP, Huber LM, et al. Predictors of pericardial effusion after orthotopic heart transplantation. J Thorac Cardiovasc Surg. 2002; 124(5): 979-983, indexed in Pubmed: 12407382. 
3. Hauptman PJ, Couper GS, Aranki SF, et al. Pericardial effusions after cardiac transplantation. J Am Coll Cardiol. 1994; 23(7): 1625-1629, indexed in Pubmed: 8195523.

4. Ciliberto GR, Anjos MC, Gronda E, et al. Significance of pericardial effusion after heart transplantation. Am J Cardiol. 1995; 76(4): 297-300, indexed in Pubmed: 7618628.

5. Almenar L, Osa A, Martínez-Dolz L, et al. Echocardiographic evaluation of the evolutionary changes after heart transplantation. Transplant Proc. 2006; 38(8): 2575-2576, doi: 10.1016/j. transproceed.2006.08.033, indexed in Pubmed: 17098007.

6. Fröhlich GM, Keller P, Schmid F, et al. Haemodynamically irrelevant pericardial effusion is associated with increased mortality in patients with chronic heart failure. Eur Heart J. 2013; 34(19): 1414-1423, doi: 10.1093/eurheartj/eht006, indexed in Pubmed: 23355650.

7. Hinderliter AL, Willis PW, Long W, et al. Frequency and prognostic significance of pericardial effusion in primary pulmonary hypertension. PPH Study Group. Primary pulmonary hypertension. Am J Cardiol. 1999; 84(4): 481-484, A10, indexed in Pubmed: 10468096.

8. Lang RM, Bierig M, Devereux RB, et al. Chamber Quantification Writing Group, American Society of Echocardiography's Guidelines and Standards Committee, European Association of Echocardiography. Recommendations for chamber quantification: a report from the American Society of Echocardiography's Guidelines and Standards Committee and the Chamber Quantification Writing Group, developed in conjunction with the European Association of Echocardiography, a branch of the European Society of Cardiology. J Am Soc Echocardiogr. 2005; 18(12): 1440-1463, doi: 10.1016/j.echo.2005.10.005, indexed in Pubmed: 16376782.

9. Billingham ME, Cary NR, Hammond ME, et al. A working formulation for the standardization of nomenclature in the diagnosis of heart and lung rejection: Heart Rejection Study Group. The International Society for Heart Transplantation. J Heart Transplant. 1990; 9(6): 587-593, indexed in Pubmed: 2277293.

10. Anderson JR, Cain KC, Gelber RD, et al. Analysis of survival by tumor response. J Clin Oncol. 1983; 1(11): 710-719, doi: 10.1200/ JC0.1983.1.11.710, indexed in Pubmed: 6668489.

11. Andersen PK. Repeated assessment of risk factors in survival analysis. Stat Methods Med Res. 1992; 1(3): 297-315, doi: 10.1177/096228029200100305, indexed in Pubmed: 1341662.

12. Team RC. R: A Language and Environment for Statistical Computing. R Foundation for Statistical Computing. 2016.

13. Al-Dadah AS, Guthrie TJ, Pasque MK, et al. Clinical course and predictors of pericardial effusion following cardiac transplantation. Transplant Proc. 2007; 39(5): 1589-1592, doi: 10.1016/j. transproceed.2006.11.014, indexed in Pubmed: 17580195.

14. Valantine HA, Hunt SA, Gibbons R, et al. Increasing pericardial effusion in cardiac transplant recipients. Circulation. 1989; 79(3): 603-609, indexed in Pubmed: 2645065.
15. Sun JP, Abdalla IA, Asher CR, et al. Non-invasive evaluation of orthotopic heart transplant rejection by echocardiography. J Heart Lung Transplant. 2005; 24(2): 160-165, doi: 10.1016/j. healun.2003.11.400, indexed in Pubmed: 15701431.

16. Jemielity M. EuroSCORE is a Predictor of Postoperative Pericardial Effusion following Heart Transplantation. Ann Transplant. 2015; 20: 193-197, doi: 10.12659/aot.892582.

17. Lund LH, Edwards LB, Kucheryavaya AY, et al. The Registry of the International Society for Heart and Lung Transplantation: Thirty-second Official Adult Heart Transplantation Report--2015; Focus Theme: Early Graft Failure. J Heart Lung Transplant. 2015; 34(10): 1244-1254, doi: 10.1016/j.healun.2015.08.003, indexed in Pubmed: 26454738.

18. Committee. NYHAC. Nomenclature and criteria for diagnosis of diseases of the heart and great vessels / the Criteria Committee of the New York Heart Association. 9th Edition ed: Little, Brown, Boston 1994.

19. Rumsfeld JS, Havranek E, Masoudi FA, et al. Cardiovascular Outcomes Research Consortium. Depressive symptoms are the strongest predictors of short-term declines in health status in patients with heart failure. J Am Coll Cardiol. 2003; 42(10): 1811-1817, indexed in Pubmed: 14642693.

20. Edelmann F, Stahrenberg R, Gelbrich G, et al. Contribution of comorbidities to functional impairment is higher in heart failure with preserved than with reduced ejection fraction. Clin Res Cardiol. 2011; 100(9): 755-764, doi: 10.1007/s00392-011-0305-4, indexed in Pubmed: 21416189.

21. Lee TT, Chen J, Cohen DJ, et al. The association between blood pressure and mortality in patients with heart failure. Am Heart J. 2006; 151(1): 76-83, doi: 10.1016/j.ahj.2005.03.009, indexed in Pubmed: 16368295.

22. Fine M. Prognosis and Outcomes of Patients With Community-Acquired Pneumonia. JAMA. 1996; 275(2): 134-141, doi: 10.1001/jama.1996.03530260048030.

23. Goldhaber SZ, Visani L, De Rosa M. Acute pulmonary embolism: clinical outcomes in the International Cooperative Pulmonary Embolism Registry (ICOPER). Lancet. 1999; 353(9162): 13861389, indexed in Pubmed: 10227218.

24. Granger CB, Goldberg RJ, Dabbous O, et al. Global Registry of Acute Coronary Events Investigators. Predictors of hospital mortality in the global registry of acute coronary events. Arch Intern Med. 2003; 163(19): 2345-2353, doi: 10.1001/ archinte.163.19.2345, indexed in Pubmed: 14581255.

25. Aranda-Dios A, Lage E, Sobrino JM, et al. Sirolimus experience in heart transplantation. Transplant Proc. 2006; 38(8): 2547-2549, doi: 10.1016/j.transproceed.2006.08.075, indexed in Pubmed: 17097997. 